\title{
Russian methods and equipment for spatial vibrocompaction foundations and structures
}

\author{
Oleg P. Minaev \\ Dr., ISSMGE, Ministry of education and science of the Russian Federation, Saint-Petersburg State Polytechnical University, 29 \\ Polytechnicheskaya st., 195251 St. Petersburg, Russia
}

\begin{abstract}
Russian method for soil compaction with the help of the vibrating driver of longitudinal vibration with a spatial compactor is used throughout the world for vibratory compaction of sandy foundation soils which can be watersaturated or slightly damp or combined together. The paper provides the results of the theoretical justification on the basis of a design model and field tests of this method for deep vibratory compaction of foundations, which show its disadvantages. These disadvantages manifest themselves the most clearly during the compaction of especially thick sandy foundations ( $9 \mathrm{~m}$ and more). This paper shows that the disadvantages of the well-known vibrating driver can be eliminated or reduced dramatically when using a vibratory compactor with radial elements placed at a certain height at the lower part of its rod. This helps to improve the performance of the vibrating driver when it is used together with a reliable and economical vibratory device.
\end{abstract}

Keywords: method for vibratory compaction, deep compaction, soil liquefaction, modernized compactor, reliability, cost-efficiency

\section{Introduction}

Russian vibratory device with spring-loaded surcharge (VPP-2), its mark of origin nowadays being V-402 (V-402VCh), was first designed by Russian scientists Prof. O.A. Savinov and A.Ya. Luskin as early as in 1950 .

Despite fierce competition with modern powerful hydraulic vibratory devices mainly of foreign make, it is still produced at Ohtinsky plant of construction machines JSC in Saint-Petersburg, Russia.

Later in 1959 on the basis of the vibratory device VPP-2 at the All-Union Scientific Research Institute of Hydraulic and Sanitation Engineering Operations (VNIIGS) there was designed a spatial compactor of longitudinal vibration for deep compaction of sandy foundations

The compactor in question includes a tubular rod provided with radial elements alongside the whole of its length in order to render it a spatial structure and therefore to boost productivity by increasing the working range of the compactor.

In this case the dynamic excitation of the vibratory device in the sandy water-saturated medium causes simultaneous impact in the whole volume of the soil body within the working range of the compactor at the depth of its penetration. Under the influence of vibration the sand is compacted as a result of more compact re-laying of some part of the sand after its liquefaction.
In the course of the 1970s-1980s further research and perfection of the method for deep compaction with the given vibrating driver was carried out at VNIIGS mainly in terms of designing a more powerful vibratory device. For this purpose at this Institute there was created a comparatively heavier vibratory device VSh-1, which can operate in the vibratory as well as vibratory-hammer mode.

The main idea of the new development is to fix the spatial elements only in the lower part of the rod of the compactor at a given height of the foundation compaction [1].

The aim of the present paper is to develop a theoretical model which explains the impact of the vibratory device on the sandy water-saturated foundation soils produced with the help of the spatial compactor of longitudinal vibration; to carryout comparative theoretical research; to find experimental proofs of the effectiveness of the compaction of foundations with the help of the modernized vibratory compactor under production conditions.

\section{Theoretical Justification}

According to the research by Prof. O.A.Savinov and Prof. I.I. Blekhman [2] for granular materials the best compaction takes place when

$$
A \omega^{2}=(0,9 \div 1) g
$$


where $A$ is the amplitude of vibration; $\omega$ isthe circular frequency of oscillations; $g$ is the acceleration of gravity.

It is known that for viscous media the amplitude of vibration decreases at the $\mathrm{x}$ distance from the source of vibration according to the exponential law:

$$
A=A_{0} e^{-\frac{x}{\delta}}
$$

where

$$
\delta \approx \sqrt{\frac{2 v}{\omega}},
$$

and $v$ is kinematic viscosity of a liquid medium. Then

$$
A=A_{0} e^{-x \sqrt{\frac{\sigma}{2 v}}}
$$

We consider that the equations (2) and(2a) are also correct for the compacted water-saturated sandy medium in question if we view $v$ and $\delta$ as ratios defined experimentally.

Let us suppose that with the given at $\omega$ the distance $x=r$ from the source of vibration the amplitude of oscillation is $A=A_{r}$, where $A_{r}$, (critical amplitude of oscillation) is minimally acceptable for the complete liquefaction of the soil in the compacted zone.

Then

$$
A_{r}=A_{0} e^{-\frac{r}{\delta}}
$$

And

$$
\ln \frac{A_{r}}{A_{o}}=-\frac{r}{\delta},
$$

which leads us to

$$
\delta=-\frac{r}{\ln \frac{A_{r}}{A_{o}}}=\frac{r}{\ln \frac{A_{o}}{A_{r}}} .
$$

The amplitude of oscillation in the vicinity of the compactor can be approximately defined using the equation

$$
A_{o}=\frac{P_{o}-P^{d y n}}{m \omega^{2}}=\frac{P_{o}-\left(P_{\mathrm{fr}}^{d y n}+P_{\mathrm{tip}}^{d y n}\right)}{\left(m_{\mathrm{v}}+m_{\mathrm{comp}}\right)(2 \pi f)^{2}},
$$

where $P_{o}$ is the driving force of the vibratory device, $m$ is the mass of the vibrating element and $f$ is the frequency of its oscillation; $m_{\mathrm{v}}$ is the mass of the vibratory device; $m_{\text {comp }}$ is the mass of the compactor;
$P_{\mathrm{fr}}^{d y n} \mathrm{U}$ is $P_{\text {tip }}^{d y n}$ the force of the dynamic resistance to penetration of the vibrating element of the compactor to the friction on the lateral surface of the compactor and its frontal penetration correspondingly.

As it is known from the research by Prof. M.G. Tseitlin and Prof. V.V. Verstov, as well as from the research of the author of the present paper, during dynamic penetration of a pile into water-saturated sandy soils a decrease in the effective resistance of the soil to sliding and a temporary decline in the resistance to the penetration of the pile take place owing to shaking and excessive pore pressure. Inparticular, during vibratory penetration (extraction) the forces of dynamic resistance are 4.5-6 times lower than the corresponding static ones in water-saturated sands concerning friction on the lateral surface and 2.5-3 times lower concerning frontal penetration.

The specifications of the vibratory device V-402 necessary for the calculations are taken from] and those of the vibratory device VUUP-6 are considered according to [2].

Using the equations (1) to (7) let us calculate the assumed amplitudes and the compaction zone by the penetration depth of the compactor designed by VNIIGS, which is driven by the vibratory device VPP-2, depending on the resistance to friction on the lateral surface of the compactor and frontal resistance to its penetration. The figures received (see Figure 1) indicate that at the compaction depth of $6 \mathrm{~m}$

the compaction zone around the compactor increases from 3 up to $4.06 \mathrm{~m}$ when the compactor approaches the surface of the foundation soil.

Let us do similar calculations for the vibrating driver with the modernized compactor whose spatial elements are decreased by three and create a curve of the compaction zone for the modernized vibratory compactor.

Let us increase the compaction depth by 2 (up to $12 \mathrm{~m}$ ) and do the calculations for the modernized compactor when the height at which the spatial elements are placed at the lower part of the rod of the compactor also equals one third of the compaction depth (4 m).

The data received are shown on the curve (Figure 2). It is easy to notice that the compaction depth for the VNIIGS vibrating driver increases from $6 \mathrm{~m}$ up to $9.53 \mathrm{~m}$ when the modernized compactor is used, the size of the compaction zone being the same $(3 \mathrm{~m})$ for both types of the compactors, as well as other parameters.

Besides, the modernized compactor allows increasing the compaction zone up to $12 \mathrm{~m}$ and more. Thus, the received by calculation compaction zone of $2.58 \mathrm{~m}$ at the depth of $12 \mathrm{~m}$ (Figure 1) can be considered acceptable for effective usage of the vibratory device at the given depth of the compaction of foundation soils. 
The calculations for the vibrating driver designed by VNIIGS show that the diameter of the compaction zone decreases up to $1.8 \mathrm{~m}$ at the depth of $9 \mathrm{~m}$, which is less than the necessary minimum (according to VNIIGS) of $2 \mathrm{~m}$ (Figure 1). Moreover, it is impossible for the compactor of this design to penetrate the depth of $12 \mathrm{~m}$.

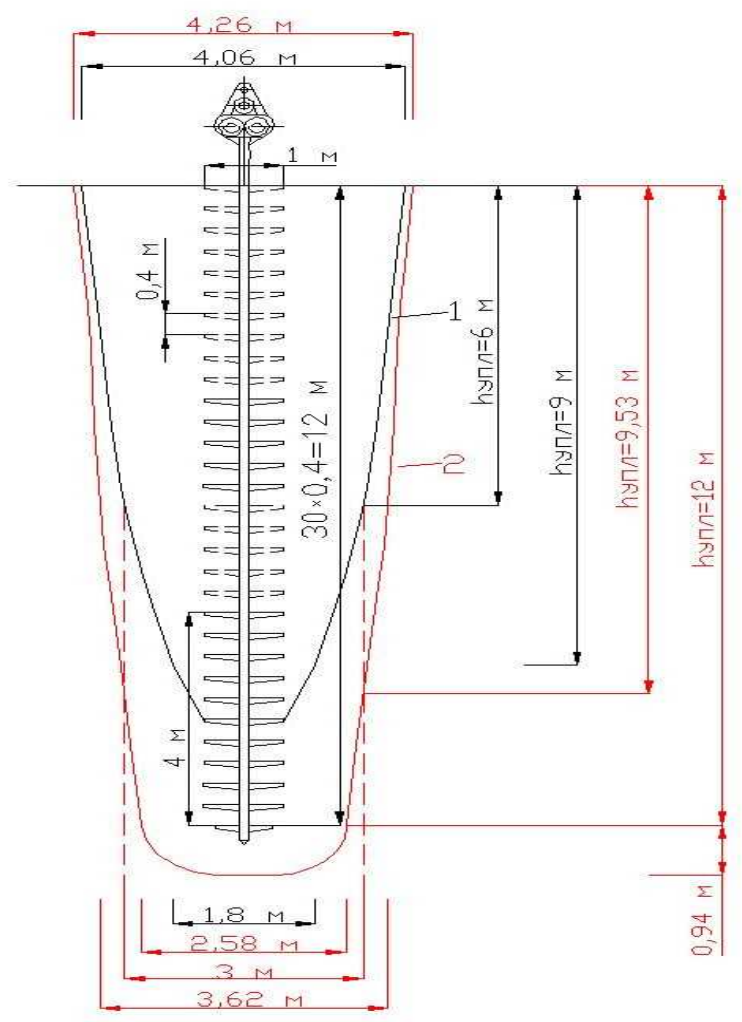

Figure 1.The compaction zone of water-saturated sandy foundation soils of medium fineness at the depth of $12 \mathrm{~m}$ produced by the 3 odernized vibratory device (1) and the decrease of the compaction zone and the depth of compaction for the VNIIGS compactor (2).

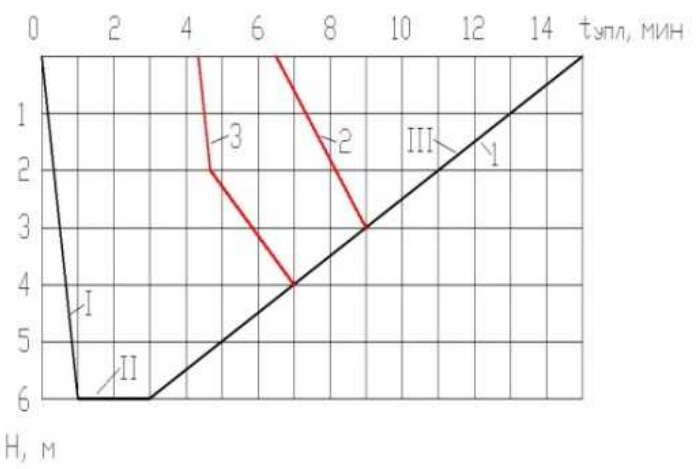

Figure 2 Graphs of foundation soils exposure to vibration: 1by the VNIIGS vibrating driver; 2 and 3 by the modernized vibratory device when the spatial elements are placed at the lower part of the rod of the compactor at the height equaling one second and one third of the compaction depth; I - the penetration stage of the compactor; II - the exposure at the given compaction depth; III - the extraction stage of the compactor.
In order to do comparative research of the attained soil density let us use as fundamental the graph of the exposure time of the vibrating driver designed by VNIIGS, as well as time graphs (made on its basis) for the modernized vibrating driver shown in Figure 2.

\section{Experimental Tests}

Initially during the construction of the Flood Protection Barrier in Saint-Petersburg there was tested the vibrating driver constructed at VNIIGS, which includes the vibratory pile driver VSh-1 and the 11.2 $\mathrm{m}$ in length compactor made of heavy-gauge pipe 113 $\mathrm{mm}$ in diameter to which metal ribs are welded lengthwise at a pitch of $500 \mathrm{~mm}$. Taking into account those ribs, the diameter of the spatial compactor is $700 \mathrm{~mm}$. To make the penetration easier the tubular rod was provided with a cross-shaped cap at its sharp point. The water was supplied to the pipe socket by a pump with maximum productivity rate of $300 \mathrm{~m} 3 / \mathrm{h}$ and head of $90 \mathrm{mH} 2 \mathrm{O}$; the flap installed in the water induction system helped to regulate the abovementioned parameters.

A crane of 25 tons load-lifting capacity was used as the basic machine.

The test compaction was carried out on fine and medium foundation sands of the already made earthen dam up to the indication of approximately $4 \mathrm{~m}$ over zero water level. The depth of underwater sand filling was $5 \mathrm{~m}$.

As a result of experimental research it was ascertained that during penetration/extraction of the compactor there appeared a crater around it, which was 0.8-1.2 $\mathrm{m}$ deep and 1.5-2 $\mathrm{m}$ in diameter.

The data of the static penetration test showed that in general the soil was compacted within a radius of 2 $\mathrm{m}$ from the centre of the compactor at the depth of up to $9 \mathrm{~m}$.

The most significant compaction of watersaturated sands below zero water level took place in the vicinity of the compaction centre, whereas it decreased already at the distance of $0.5 \mathrm{~m}$ from it. At the same time there happened a washout in the central zone, i.e. loosening of the surface layer of the soil up to the depth of 1.5-2 $\mathrm{m}$ and further under the water head when the compactor was extracted. The attempt to stop the water supply during the extraction of the compactor from sandy foundation soil led to its blocking up.

However, during further compaction it was discovered that the vibrating driver was rather unreliable due to frequent breakdowns of the vibratory device. That was why the construction of the compactor was altered and the modernized vibrating driver was used in further experimental works of soil foundation compaction at the same part of the dam. 
The results of the comparative field observations were obtained for both the vibrating driver designed by VNIIGS and the modernized vibratory compactor whose upper horizontal ribs had been cut off leaving them only in the section of 3.5-4.5 $\mathrm{m}$ in the lower part of the rod. According to theoretical research, this change in the construction is supposed to alleviate the work of the vibrating device, because the horizontal ribs do not have to vibrate and penetrate the upper part of the damp soil, while the rest of the ribs are to preserve the compacting ability in the area where water is supplied.

The tests of the modernized vibratory compactor with the vibrating device V-401 were carried out with the interval of penetration being $3 \times 3$. The crane used had a significantly lower load-lifting capacity.

According to the static penetration test the depth of the compaction was mainly $9 \mathrm{~m}$. At the same time the soil was more homogeneously compacted in the central part, as well as within the radius of $1.5 \mathrm{~m}$. In addition, the soil was not loosened in the central zone around the compactor.

The above-mentioned can be clearly confirmed by the comparison of the static penetration tests data along the penetration axis shown in Figure 3.It is easy to notice that before the compaction the resistance to the penetration of the sharp tip of the penetrometer $q$ p in the above-water zone varied from 3.5 up to $8 \mathrm{MPa}$ and in the underwater zone it was $2.5-8 \mathrm{Mpa}$. After the compaction done with the help of the VNIIGS vibrating driver the $q \mathrm{p}$ data in general varied from 1.5 to $25.5 \mathrm{Mpa}$ in the above-water zone and from $6.5 \mathrm{up}$ to $25.5 \mathrm{Mpa}$ in the underwater zone. At the same time the increase of $q \mathrm{p}$ in the underlying layer of the foundation did not exceed $6.5 \mathrm{Mpa}$ and there was a decrease of $q \mathrm{p}$ from 3.5-5.5 up to $1.5-3 \mathrm{Mpa}$ at the depth of up to $2 \mathrm{~m}$ from the foundation surface.

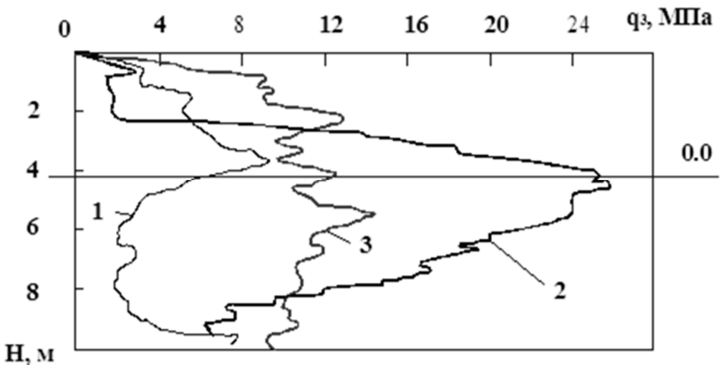

Figure 3.The comparative data of the static penetration tests of the foundation: 1 - before the compaction, 2, 3 - after the compaction done with the vibrating driver designed by VNIIGS (2) and of modernized construction (3).

According to the static penetration test (see Figure 3 ), after the compaction done with the modernized vibrating driver the resistance to the penetration of the sharp tip of the penetrometer $q \mathrm{p}$ increased generally up to 9-14 Mpa alongside the whole depth of the compacted layer of the foundation. The data received for $q p$ characterize fine and medium sands as medium and dens]. This provides resistance of sandy foundation soils of buildings and structures to most dynamic and seismic loads [3-5].

The vibrating driver with the modernized compactor driven by the vibrating device VPP-2 was used to compact the water face of the dam in the motorway bed as well as the sandy ballasts of the floating gates of the navigation pass and other objects during the construction of the Flood Protection Barrier (FPB) in Saint-Petersburg. The volume of the compacted soil was not less than $1.0 \mathrm{mln}$ cub. M merely during the FPB construction.

\section{Conclusions}

1. Russian vibratory device and the method for the compaction of foundations with the help of the vibrating driver of longitudinal vibration with the spatial compactor is widely used to compact sandy foundation soils, which can be water-saturated or slightly damp, as well as combined together.

2 . The disadvantages of the given vibrating driver can be eliminated or decreased significantly if a compactor with radial elements placed at a given height in its lower part is used.

3. The field testing and experimental compaction under production conditions confirmed that the given solution enables not only to provide easier penetration of the compactor into foundation soils and its vibratory extraction, but also to preserve the compacting capacity of the compactor, as well as to improve the uniformity of soil foundation compaction alongside the whole targeted depth of compaction.

4. The present-day building practices enable to recommend the vibratory device V-402 with the modernized compactor for the compaction of sandy soils of foundations and structures when the layer to be compacted varies from $3 \mathrm{~m}$ to $12 \mathrm{~m}$ in different parts of the world.

\section{REFERENCES}

1) Minaev, O.P. (2011): Development of vibratory method for soil compaction during construction, Soil Mechanics and Foundation Engineering, 48 (51), 90-195.

2) Blekhman, I.I., (2013): The theory of vibratory processes and devices, Vibration mechanics and vibratory equipment. Monograph. St. Petersburg, Russia, Ore and Metals publishing house, 640 .

3) Ivanov, P.L. (1980): Consolidation of Saturated Soils by Explosions, International Conference on Compaction. Paris, 1,. 331-337.

4) Seed, H.B., Idriss, I.M. (1982): Ground motions and soil liquefaction during earthquakes. Monograph.. USA, Oakland , C.A.: Earthquake Engineering Research Institute, 320.

5) Ishihara, K. (2006): Soil Behaviour in Earthquake Geotechnic, Monograph. USA, Oxford, Clarendon Press: Department of Civil. Engineering Science University of Tokyo, 384 . 